\title{
Defect detection based on two different algorithms of analysis and
}

\section{comparison}

\author{
Zhang Xiaowei $^{1, a^{*}}$, Fan Xiujuan ${ }^{2, b}$ \\ ${ }^{1}$ Chaoyang district, Beijing, China Beijing Institute of Fashion Techology \\ ${ }^{2}$ Chaoyang district, Beijing, China Beijing Institute of Fashion Techology \\ a602256515@qq.com, b gxyfanxi@bift.edu.cn
}

Keywords: Hilbert-Huang transform; Fabric defect detection; cross-correlation; OpenCV

Abstract. Fabric defect detection has been an active area of research since a long time and still a robust system is needed which can fulfill industrial requirements. A robust automatic fabric defect detection system (FDDS) would results in quality products and more revenues. Many different approaches and method have been tried to implement FDDS. This paper presents a new scheme for automated FDDS implementation using Hilbert-Huang Transform (HHT) and also compares it with OpenCV approach. In my implementation of both approaches in same environment, the HHT approach produces higher defect detection accuracies than OpenCV approach and more computationally efficient. The article is divided into four parts, the first part is the introduction of two methods, the second part for the two methods defect detection system implementation, the third part is the results and the discussion of two methods, the fourth part is the conclusion.

\section{Introduction}

\section{HHT}

HHT is used to deal with nonlinear and non-stationary signal, the method of its adaptive is good, can be used to analyze all kinds of signals. Compared with the Fourier transform, the HHT has more explicit time-frequency description; filter performance is even more acute. In addition, its implementation is simple and can be real-time calculation. Therefore, for the engineering application and theory research, HHT has very important significance. HHT method has two parts: Empirical mode decomposition and Hilbert spectrum analysis.

\section{Empirical mode decomposition (EMD)}

Empirical mode decomposition is a complex series of non-stationary and nonlinear signals is decomposed into a set of linear and stationary sequence of intrinsic mode function (IMF), The sequence contains the local characteristics of original signal in different time scale. Algorithm is as follows:

Search the maximum and minimum number of the primary signal, then a cubic saline interpolation method is used all the maximum formation on the envelope and minimum envelope to get the original signal envelope.

Averaging fluctuation of the signal envelope, get the average $\mathrm{m}_{1}(\mathrm{t})$.

Subtract $m_{1}(t)$ from the original signal, get the IMF's a prototype function $h_{1}(t)=x(t)-m_{1}(t)$, Determine whether $h_{1}(t)$ meet the EMD decomposition conditions, If you don't meet, Will continue to repeat the steps until the $h_{1}(t)$ as the original signal met, After k times, until meet the conditions 
of the IMF's weight, as $\mathrm{c}_{1}(\mathrm{t})$

The original signal Minus the $\mathrm{c}_{1}(\mathrm{t})$ get the residual signal $\mathrm{r}_{1}(\mathrm{t}), \mathrm{r}_{1}(\mathrm{t})$ is the residual, $\mathrm{r}_{1}(\mathrm{t})$ as a new signal , continue and repeat the above steps, get the second IMF component $c_{2}(t)$, In turn, get the third, the fourth...the nth, until the resulting residual component $\mathrm{r}_{\mathrm{n}}(\mathrm{t})$ into a constant or monotonic function, the decomposition is completed.

Merger of each component, The original signal can be expressed as:

$$
x(t)=\sum_{i=1}^{n} c_{i} t \rightarrow r_{n} t(
$$

Among these, $r_{n}(t)$ is residual component, because $r_{n}(t)$ is a trend item and constant, this practice can be ignored.

\section{HHT Time spectrum and marginal spectrum}

According to the result of empirical mode decomposition, By Hilbert transforming the Each intrinsic mode function component $c_{i}(t)$ :

$$
\hat{c}_{i}(t)=\frac{1}{\pi} P \int \frac{c_{i}(\tau)}{t-\tau} d \tau
$$

Among these, $\mathrm{P}$ is Cauchy principal value. The analytic signal $E_{i}(t)$ is Made up of $c_{i}(t)$ and $\hat{c}_{i}(t)$, it is:

$$
E_{i}(t)=c_{i}(t)+j \hat{c}_{i}(t)=a_{i}(t) e^{j \varphi_{i}(t)}
$$

In the formula(3), $a_{i}(t)=\sqrt{c_{i}{ }^{2}(t)+\hat{c}^{2}{ }_{i}(t)}$ is instantaneous amplitude, $\varphi_{i}(t)=\arctan \left[\hat{c}_{i}(t) / c_{i}(t)\right]$ is instantaneous phase. So Instantaneous frequency $\omega_{i}(t)=\left[d \varphi_{i}(t) / d t\right]$, thus Each IMF can be expressed as:

$$
c_{i}(t)=\operatorname{Re}\left[a_{i}(t) e^{j \varphi_{i}(t) d t}\right]=\operatorname{Re}\left[a_{i}(t) e^{j \int \omega_{i}(t) d t}\right]
$$

Overall the Hilbert transform of original signal $x(t)$,Can get $x(t)$ on the time-frequency representation:

$$
x(t)=\operatorname{Re} \sum_{i=1}^{n} a_{i}(t) e^{j \int \omega_{i}(t) d t}
$$

By the formula is, the amplitude and frequency of original signal $x(t)$ are all about the function of time. So we can make the amplitude and frequency change over time in a three-dimensional figure performance. Thus formed the Hilbert amplitude spectrum, as $H(\omega, t)$. Integral of the Hilbert spectrum can get the definition of the Hilbert marginal spectrum: (let it as $h(\omega))$

$$
h(\omega)=\int_{0}^{T} H(\omega, t) d t
$$


The EMD decomposition to the original signal, had a theoretical intrinsic mode function containing single frequency components, benefits of processing the signal after decomposition. But in the process of decomposition, ignore the endpoint effect and envelope method choice, so there will be contained false frequency components of the IMF's intrinsic mode function. For such signals, only from the spectrum diagram is unable to accurately identify whether contained false composition, and it is hard to identify the periodic signal. So in order to determine the similarity between different spectrum, it is need to do cross correlation with frequency spectrum, Marginal spectrum of cross-correlation function is defined as:

$$
R_{H_{1} H_{2}}(\omega)=\lim _{W \rightarrow \infty} \int_{0}^{T} H_{1}(\omega) H_{2}(\omega+W) d \quad a
$$

For its discrete form:

$$
R(\omega)=\sum_{w} H_{1}(\omega) H_{2}(\omega+w)
$$

We can use the formula (7) to calculate the cross-correlation between different marginal spectrum number, the degree of cross-correlation between marginal spectrum is obtained. In order Cross-correlation number to determine the correlation between different marginal spectrum.

In general, normal fabric image signal on the spectrum reflection should be continuous cyclical, and each cycle of similarity is very high. Because the existence of defects affect the cyclical nature of spectrum, in some specific area curve will mutate. In this article, the cross-correlation function describes marginal spectrum frequency $\omega$ and $\omega+W$ related degree between the different time. It is the measurement for similarity between marginal spectrum, reaction is the similarity between different marginal spectrum. Cross-correlation function of the correlation coefficient of similar size can be used as a marginal spectrum measurement, can directly reflect the similarity between marginal spectrum. But for the correlation coefficient size represents meaning the definition of the boundary is not consistent in academia at present, according to the experience, usually using the correlation coefficient $(0-1)$ to show the related degree, the greater the value, the lower the similarity degree.

\section{OpenCV method}

OpenCV is: the full name of the Open Source Computer Vision Library. OpenCV is a issue of the cross-platform based computer vision library; you can run on Linux, Windows and Mac OS operating system. It's lightweight and efficient and it made up of a series of $\mathrm{C}$ function and a small amount of $\mathrm{C}++$ class, at the same time provides the Python, Ruby, MATLAB language interface; realize the many general algorithm of image processing and computer vision.

In this paper using OpenCV data structure and the commonly used image process ing method, the paper puts forward a kind of new fabric defect detection system development platform, use this environment, to improve the existing algorithm of fabric defect, and OpenCV Visual library, developed the fabric defect detection system with high efficiency System. Compared with the Matlab, generic C or C + + platform. Defect detection system development cycle based on OpenCV is short, the program run faster and defect detection accuracy. 


\section{Defect detection system imple mentation}

\section{HHT method}

Turn two-dimensional images to one-dimensional signal

1) Access the images (two-dimensional signal) and according to the type (9) as matrix form

2) On the basis of the above (9) turn two-dimensional signals by row (column) to the onedimensional signal, and get the one-dimensional image results, As shown in figure 2.

$$
A=\left[\begin{array}{ccccc}
a_{11} & a_{12} & \ldots & a_{1 n} \\
a_{21} & a_{22} & \ldots & a_{2 n} \\
a_{31} & a_{32} & \ldots & a_{3 n} \\
\ldots & \ldots & \ldots & \ldots \\
a_{n 1} & a_{n 2} & \ldots & a_{n n}
\end{array}\right] \rightarrow X=\left\{\left[a_{1 i}\right],\left[\begin{array}{ll}
a_{2} \\
2
\end{array}\right],\left[a_{i}\right] \cdots\left[a_{n i}\right]\right\}, i=1,2,3 \ldots
$$

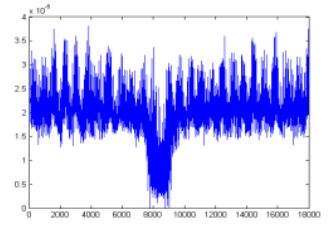

Figure 1: Defect image merge results

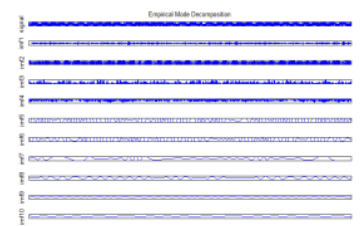

Figure 2 : Some EMD decomposition results

One-dimensional signal of Hilbert marginal spectrum

1) The above combined one dimensional signal $X(t)$ the EMD decomposition, The result is shown in figure2: The picture above shows the EMD decomposition of the IMF1, IMF2 ,IMF3... etc. Component and meet the EMD decomposition conditions.

2) All components of the EMD decomposition after the Hilbert transform, get the Hilbert spectrum(figure3) and the Hilbert marginal spectrum (figure4), as shown in the figure below:

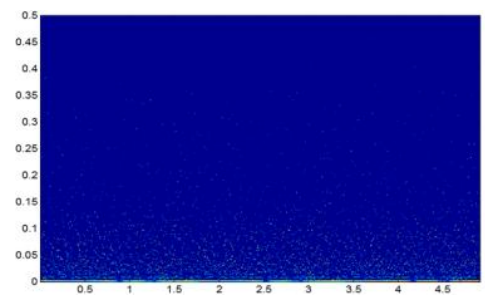

figure 3 HHT spectrum

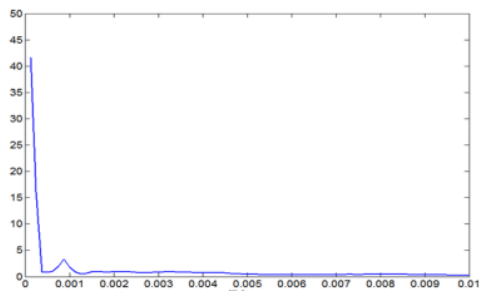

figure 4 HHT marginal spectrum

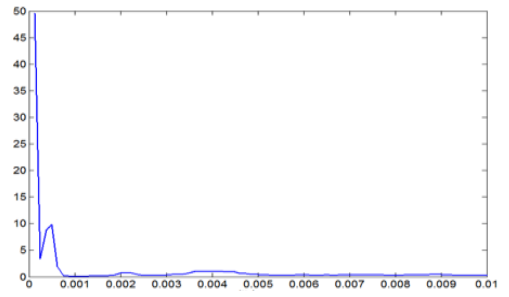

figure 5 marg inal spectrum of no defects

3) Selecting figure (7) b without defect template you repeat the steps to get the defect image of marginal spectrum is shown in figure 5:

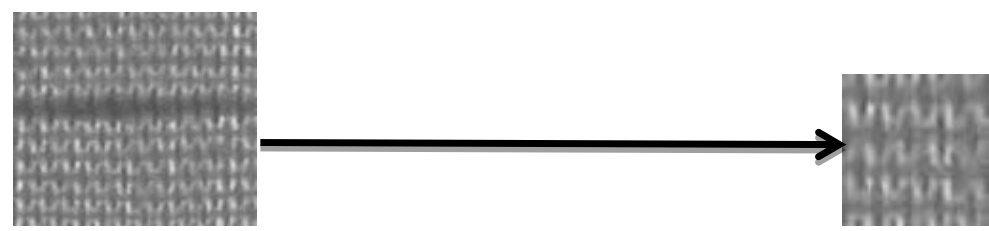

(a) Contain the defect fabric images

(b) Defect-free template image

figure 6 Containing defect fabric image and the template

To get a defect image marginal spectrum and defect-free marginal spectrum template image cross-correlation, as shown in figure 7 


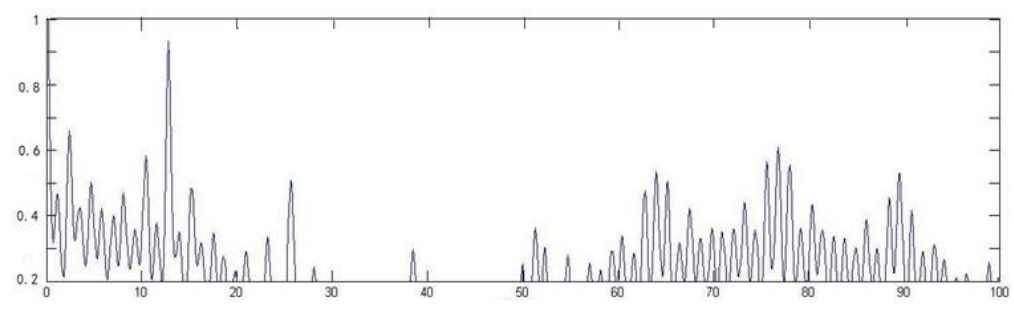

figure 7 the cross-correlation results

By above knowable, figure 6 defect-free image template and have defects in marginal spectrum after cross-correlation, the number of relationships to each other distribution between 0 and 1. According to the theory, exists defect on the number of regional relations with each other, the similar degree is low, no relationship to each other for small defect of the area, the similarity degree is high. But from the figure 7 can only know each other only roughly range value, In order to further get cross-correlation values and validation algorithm is effect, still need to do the simulation experiment.

\section{OpenCV method}

As an example to broken warp and Weft defects, the following is some pictures in the experiment, the image is processed in figure 8 , in order to get the characteristics of the defect images, first CI normal distribution should be adopted to get double threshold, then let the gray image processing using CV-threshold function, the result is shown in figure 9:

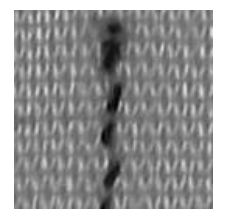

broken warp weft defects figure 8

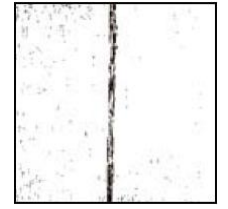

broken warp Weft defects

Figure9

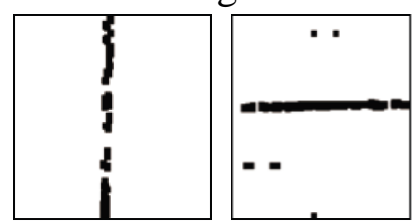

broken warp weft defects

figure 10

In figure9, there will be a lot of noise in the defect images, The noise can be removed with arithmetic average filtering or corrosion function, The results are shown in figure 10:

Noise is processed, there are still some images of the isolated points, these points are almost small defect. The idea of handling these defect is: firstly, the outlier noise removal of less than a fixed size, the defect of bigger than most of these points is around it and it will participate in calculation. Circumference gets rid of the noise based on the perimeter. Isolated point of connection will need to call cvgetreal2d and cvsetreal2d two functions, in the image and change the pixel values of the pixels of the image of the visit, the results shown in figure 11:

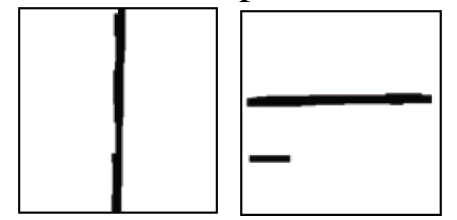

broken warp weft defects figure 11

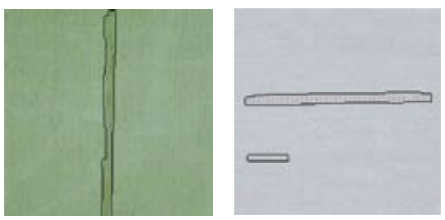

broken warp weft defects

figure 12

Last call cvCanny edge detection function for edge detection of defect image of figure 11 linearization, then call cvCopy function in the original color defect image sketch an outline of the 
defect, defect location, the results are shown in figure 12.

\section{Results and discussion}

\section{HHT method}

Choose a standard template image. In order to detection algorithm, here are three template image

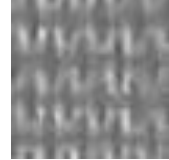

template image a

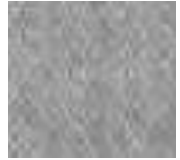

template image $b$

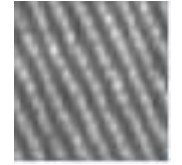

template image $\mathrm{c}$

Window segmentation for fabric image to be detected.

In order to achieve the ideal effect of detection, the fabric image will be divided into 9 images based on experience value, and each image is $128 * 128$ pixels, segmentation results are shown in figure 13 below:

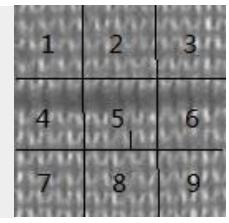

image a)

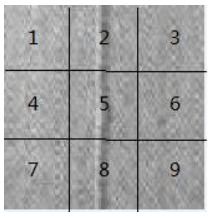

image b)

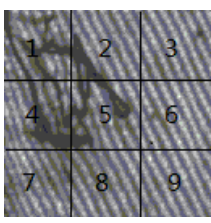

image c)

figure 13 The fabric image window segmentation results

Standard template image and put marginal spectrum of every split window into cross-correlation.

The template image and the marginal spectrum of each window after cross-correlation according to the formula(7), The results as shown in chart 1 :

chart1

\begin{tabular}{cccccccccc}
\hline Number of subimage & $\mathbf{1}$ & $\mathbf{2}$ & $\mathbf{3}$ & $\mathbf{4}$ & $\mathbf{5}$ & $\mathbf{6}$ & $\mathbf{7}$ & $\mathbf{8}$ & $\mathbf{9}$ \\
\hline Template a and images a & 0.91 & $\mathbf{0 . 7 9}$ & 0.93 & 0.93 & $\mathbf{0 . 8 1}$ & 0.95 & 0.92 & $\mathbf{0 . 8 0}$ & 0.94 \\
Template b and images b & 0.95 & 0.97 & 0.96 & $\mathbf{0 . 7 9}$ & $\mathbf{0 . 8 0}$ & $\mathbf{0 . 8 0}$ & 0.93 & 0.94 & 0.93 \\
Template c and images c & $\mathbf{0 . 7 6}$ & $\mathbf{0 . 7 9}$ & 0.90 & $\mathbf{0 . 8 0}$ & $\mathbf{0 . 8 1}$ & 0.92 & 0.96 & 0.93 & 0.92 \\
\hline
\end{tabular}

As you can see from the form above results, Defect area is divided into the picture with the template image on the number of relationships to each other Significantly greater than defect-free area window images, This is consistent with the result we expected, The lower the correlation coefficient, the greater the degree of similarity, in line with the theoretical predictions 。 Because of the existence of defects destroys the normal fabric texture, Which leads to the difference between defect signals and normal fabric。In order to verify the correctness of the theory we get results average correlation coefficient of 0.80 in the table as a threshold to identify defects, the defect in the form of binary image extracted from the background The simulation results are as follows:

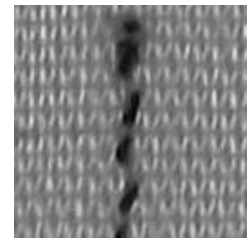

a) broken warp

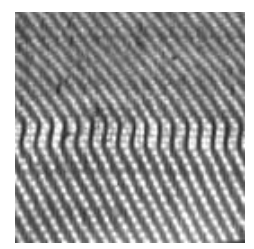

b ) missing weft

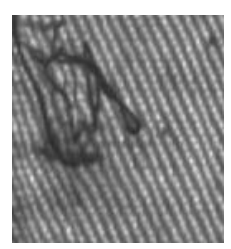

c) broken hole

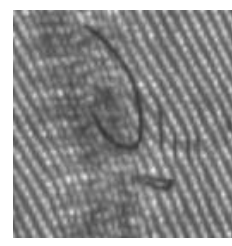

d) hook silk

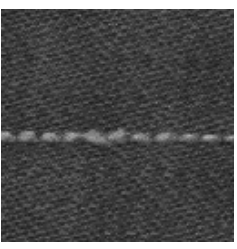

e) Lack of weft

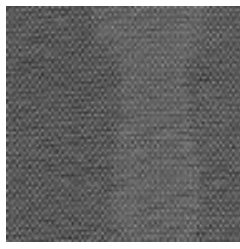

f) coarse end

figure 14 contain the defect fabric images 


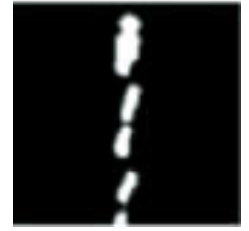

a) broken warp

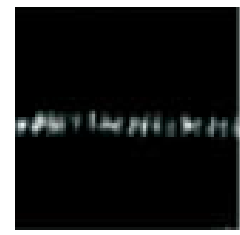

b) missing weft

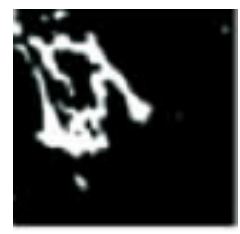

c) broken hole

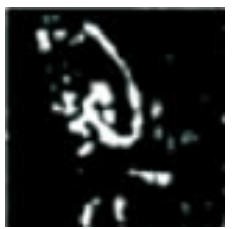

d) hook silk

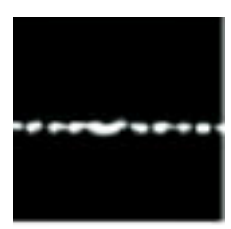

e) Lack of weft

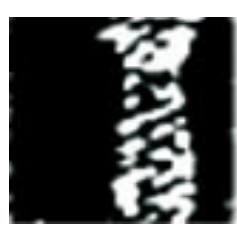

f) coarse end

figure 15 HHT method defect detection results

From the simulation results, Defect identification is more effective, Defect area can be identified basically, And the boundaries obvious. Experiments show that the algorithm presented in this paper to test the different kinds of defects, the detection of high efficiency and good adaptability.

\section{OpenCV method}

OpenCV method is used for the same fabric image containing defect detection, Matlab simulation test results are as follows:

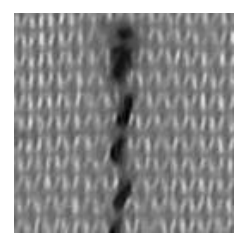

a) broken warp

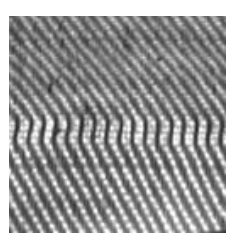

b) missing weft

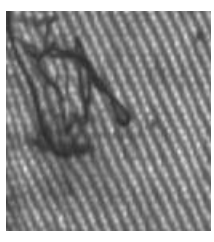

c) broken hole

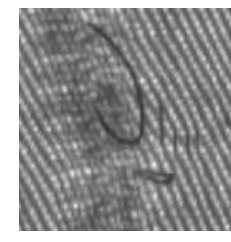

d) hook silk

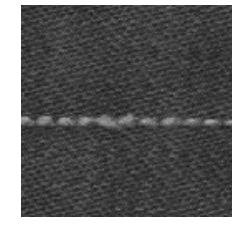

e) Lack of weft

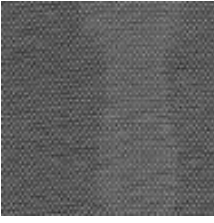

f) coarse end figure 16 contain the defect fabric images

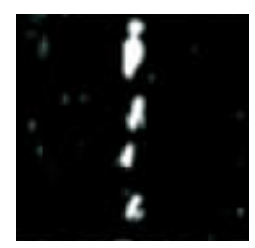

a) broken warp

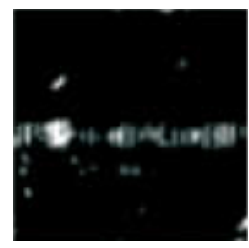

b) missing weft

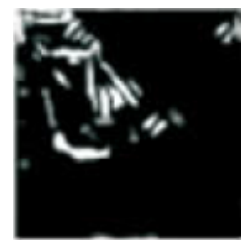

c) broken hole

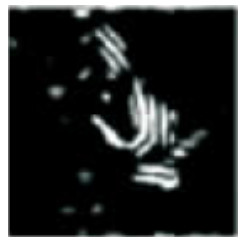

d ) hook silk

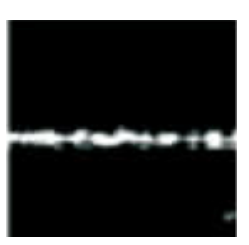

e) Lack of weft

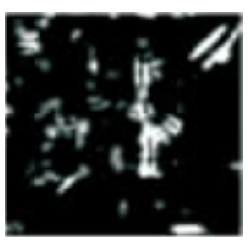

f) coarse end

figure 17 Open CV method defect detection results

\section{Conclusion}

In general, defect detection approaches can be classified into two categories: supervised and unsupervised detection approach. Practically a conventional supervised approach based on the knowled ge of some particular defect types may not be suitable. On the other hand, the design of an unsupervised approach is rather complicated. In this paper, two unsupervised defect detection methods have been implemented and compared.

The figure 15 clearly show that most of the defects can be successfully detected. These excellent results clearly indicate that the proposed schemes (HHT) are an effective and robust means for detecting fabric defects. According to the figure 15 and 17, HHT method show better results than OpenCV method.

Sources of funding: Beijing Institute of Fashion Techology Innovation team project Item no.: pttbifd_002 


\section{References}

[1] Bu H G,Huang X B,Wang J,et al.Detection of fabic defects by auto-regressive spectrul analysis and support vector data description[j].Text Res J.2010,80:579-589.

[2] HU M C,TSAI I S.Fabric inspection based on best waveletpacket bases [J].Textile Research Journal,2000,70(8):662- 670.

[3] Yang Ya Xue Yuncan, ShaWei JiLing. Based on orthogonal wavelet decomposition of fabric defect detection [J]. Microcomputer processing, 2015,(07)

[4] $\mathrm{Wu}$ Yulian, feng xiangchu. Balance the regularization of cartoon texture non-convex image restoration [J]. Journal of xi'an electronic science and technology news. 2014 (05)

[5] Huang Cheng TI. Hilbert huang transform and its application research. Xi 'an: xi 'an jiaotong university, a master's degree thesis.

[6] Li Wenyu long-di cheng. Fabric defect detection based on machine vision and image processing methods. Journal of textile [J], 2014, 35 (3) : 159-163.

[7] Feng-qin yu Zhao jing, Sunyan. More direction of fabric defect edge detection based on Contourlet transform [J]. Computer engineering and application, 2012 ,48(27):194-168. 